\title{
Study of saline wastewater influence on activated sludge flocs through automated image analysis
}

\author{
Daniela P Mesquita, ${ }^{a}$ Antonio L Amaral, ${ }^{a, b}$ Eugenio C Ferreira ${ }^{a}$ and \\ Maria AZ Coelhoc*
}

\begin{abstract}
BACKGROUND: In activated sludge systems, sludge settling ability is considered a critical step in effluent quality and determinant of solid-liquid separation processes. However, few studies have reported the influence of saline wastewater on activated sludge. This work aims the evaluation of settling ability properties of microbial aggregates in a sequencing batch reactor treating saline wastewaters of up to $60 \mathrm{~g} \mathrm{~L}^{-1} \mathrm{NaCl}$, by image analysis procedures.

RESULTS: It was found that the sludge volume index (SVI) decreased with salt content up to $20 \mathrm{~g} \mathrm{~L}^{-1}$, remaining somewhat stable above this value. Furthermore, it was found that between the first salt concentration $\left(5 \mathrm{~g} \mathrm{~L}^{-1}\right)$ and $20 \mathrm{~g} \mathrm{~L}^{-1}$ aggregates suffered a strong deflocculation phenomenon, leading to a heavy loss of aggregated biomass. Regarding SVI prediction ability, a good correlation coefficient of 0.991 between observed and predicted SVI values was attained.

CONCLUSION: From this work the deflocculation of aggregated biomass with salt addition due to pinpoint floc formation, dispersed bacteria growth and protozoa absence could be established. With respect to SVI estimation, and despite the good correlation obtained, caution is advisable given the low number of SVI data points.

(c) 2008 Society of Chemical Industry
\end{abstract}

Keywords: settling ability; image analysis; activated sludge; aggregates; multivariate statistical techniques

\section{INTRODUCTION}

The desalination step in petroleum processing and other industrial activities such as fish processing and chemical manufacturing generates high-saline wastewaters that interfere with biological treatment efficiencies and with population structure. ${ }^{1}$ The sequencing batch reactor (SBR) is one of the most interesting activated sludge processes used for saline wastewater treatment recently studied by several researchers. ${ }^{1-4}$ Salt effects on the performance of biological processes are extremely important and have been carefully studied as they are known to have an adverse impact on the performance of a typical activated sludge system and on biological nutrient removal., ${ }^{5,6}$ As a matter of fact, high salt levels lead to a less diverse microbial population and could have a negative impact on sludge settling characteristics. ${ }^{1}$ The improper formation of activated sludge aggregates causes poor settling, resulting in high effluent turbidity and, consequently, lower clarifier efficiency. ${ }^{6}$ Therefore, it comes as no surprise that saline wastewaters have been reported to result in unstable sludge blankets in the settler and low separation efficiency. ${ }^{7}$ The solid-liquid separation in wastewater treatment plants (WWTPs) is commonly assessed through sludge volume index (SVI) evaluation, characterizing the sludge settling ability. ${ }^{8}$ However, as the SVI only provides a macroscopic evaluation of the activated sludge, the microscopic characteristics of the sludge should be also determined by examination under an optical microscope.
Nowadays, microscopic image analysis procedures are mainly used for morphological characterization of microbial aggregates and evaluation of filamentous bacterial content in an attempt to correlate such parameters with biomass settling ability properties. ${ }^{9,10}$ Furthermore, in recent years, activated sludge characterization by means of automated image analysis has been used to monitor bulking events in pilot plants. ${ }^{11-13}$ In fact, successful image processing methodologies have already been used to provide morphological data further processed by partial least squares regression (PLS). ${ }^{14}$ In that study the existence of a severe bulking problem of a non-zoogleal nature could be established, while PLS analysis revealed a strong relationship between total suspended solids (TSS) and

* Correspondence to: MariaAZCoelho, Departamento de Engenharia Bioquímica, Escola de Química, Universidade Federal do Rio de Janeiro, Rio de Janeiro 21949-900, Brazil.E-mail: alice@eq.ufrj.br

a IBB-Institute for Biotechnology and Bioengineering, Centre of Biological Engineering, Universidade do Minho, Campus de Gualtar, 4710-057 Braga, Portugal

b Instituto Superior de Engenharia de Coimbra, Instituto Politécnico de Coimbra, Rua Pedro Nunes, Quinta da Nora, 3030-199 Coimbra, Portugal

c Departamento de Engenharia Bioquímica, Escola de Química, Universidade Federal do Rio de Janeiro, Rio de Janeiro 21949-900, Brazil 
the total aggregate area, as well as a close correlation between the filamentous bacteria/suspended solids ratio and the SVI.

At present, there have been few reports on the influence of saline wastewater on activated sludge flocs, the appearance of which is a determinant factor in solid-liquid separation. The current work studied the effect of saline wastewater $\left(0-60 \mathrm{~g} \mathrm{~L}^{-1} \mathrm{NaCl}\right)$ in an SBR unit using conventional activated sludge. Structural and morphological changes of microbial aggregates were analyzed by image analysis. Furthermore, PLS analysis was employed to evaluate the SVI based on collected image analysis data.

\section{MATERIAL AND METHODS \\ Experimental setup}

Experiments were carried out in a sequencing batch reactor $(50 \mathrm{~L})$ equipped with a controllable feed pump, air supply, mechanical agitator and sensor apparatus (level, oxidation reduction potential (ORP), pH and dissolved oxygen). Synthetic wastewater was fed to the reactor at a flow rate of $5 \mathrm{~L} \mathrm{~h}^{-1}$. Between each experiment, biomass acclimation was performed over 15 days, allowing microorganisms adaptation to each salt concentration. The operating cycle lasted for $6 \mathrm{~h}$ with filling, reaction and settling phases. Based on the study of Coelho et al., the implementation of a discrete fill strategy consisting of symmetric pulses for the wastewater and oxygen supply was favoured. ${ }^{15}$ During the discrete fill, throughout a total period of $3.5 \mathrm{~h}$, sequences of aerobic and anoxic phases were performed, allowing for lower substrate levels and diminishing cell inhibition. Afterwards, the reaction step took place over $2 \mathrm{~h}$. Finally, a period of around $0.5 \mathrm{~h}$ was allowed for biomass settlement, followed by removal of the supernatant.

\section{Synthetic wastewater composition and sludge characteristics} Synthetic medium prepared with demineralized water had the following composition ( $\mathrm{mg} \mathrm{L}^{-1}$ ): $\mathrm{NH}_{4} \mathrm{Cl}, 76.1 ; \mathrm{C}_{6} \mathrm{H}_{12} \mathrm{O}_{6}$, 300; $\mathrm{MgSO}_{4} .7 \mathrm{H}_{2} \mathrm{O}, 16.7 ; \mathrm{NaHCO}_{3}, 243.3 ; \mathrm{Na}_{2} \mathrm{CO}_{3}, 162.2$; $\mathrm{Na}_{2} \mathrm{HPO}_{4} .12 \mathrm{H}_{2} \mathrm{O}, 46.2 ; \mathrm{CaCl}_{2} .7 \mathrm{H}_{2} \mathrm{O}, 4.7$; and $\mathrm{KCl}$, 4.7. For biomass maintenance, trace elements were added at lower concentrations (below $0.2 \mathrm{mg} \mathrm{L}^{-1}$ ). Salt $(\mathrm{NaCl})$ concentration varied from 0 to $60 \mathrm{~g} \mathrm{~L}^{-1}$. The sludge, generously provided by Petrobras S.A., was maintained in the reactor throughout the entire experimental period. The mixed microbial culture concentration was approximately $2.5 \mathrm{~g} \mathrm{~L}^{-1}$. During reactor operation no significant biomass growth was detected; i.e., a constant ratio between volatile suspended solids (VSS) and total suspended solids (TSS) was found. A similar situation has been described by other authors employing the same experimental setup. ${ }^{16,17}$

\section{SVI and turbidity measurements}

SVI was determined to evaluate the sludge settling properties according to Standard Methods in a $1 \mathrm{~L}$ settling cylinder after each SBR run. ${ }^{18}$ The turbidity of the effluent was measured using the supernatant through a Hach DR4000-UV spectrophotometer at $860 \mathrm{~nm}$ (Hach Co., Loveland, CO, USA) after 30 min of biomass settling.

\section{Image analysis and processing \\ Image acquisition}

The activated sludge analyzed in this work was collected from the SBR at the end of each saline test. During the course of this study, only the aggregated biomass was monitored by image analysis since free filamentous bacteria were absent from the activated sludge. For aggregates image acquisition, a volume of $50 \mu \mathrm{L}$ was placed on a slide and covered with a $24 \times 32 \mathrm{~mm}$ coverslip for visualization and image acquisition by bright-field microscopy. Around 100 images $(2048 \times 1536$ pixels) were acquired per sample through visualization on a Nikon Eclipse 200 microscope (Nikon Corp., Tokyo, Japan), with a total magnification of $40 \times$, coupled to a Nikon Coolpix 990 RGB camera.

Aggregated bacterial morphological descriptors were determined using software routines developed in ImageJ $(\mathrm{NIH}$, Bethesda, MD, USA) and Matlab 7.1 (The Mathworks, Natick, MA, USA) by Amaral. ${ }^{13}$ ImageJ was used for image processing to obtain binary images of the aggregates from the grayscale images, comprising image pretreatment, image segmentation and debris elimination. Matlab was used to determine the aggregate morphological parameters, described below.

\section{Image pretreatment}

The image pre-processing stage consisted of enhancing the grayscale images by background removal. In this stage, a background subtraction was performed by the use of a grayscale erosion operation. In this way background light differences are minimized and the aggregates are further enhanced from the background.

\section{Aggregate segmentation}

The segmentation stage comprised segmentation of the grayscale image to a binary image and the elimination of small amounts of debris. Therefore, the first step was segmentation of the grayscale image by a predefined threshold level. The resulting binary image (objects with value 1 and background with value 0) was further processed to remove small amounts of debris, discarding objects smaller than 16 pixels. This step was performed by the use of morphological erosion and reconstruction operations, so that a clean aggregate binary image was obtained. Figure 1 represents an example of original and final binary images obtained from the program.

\section{Determination of structural and morphological parameters}

Supported by the previous study of Amaral and Ferreira, several aggregate parameters were determined and are described below. ${ }^{14}$

The number of aggregates was given by the total number of aggregates not cut off by the image boundaries. The aggregate area was determined as the sum of the pixels of the projected surfaces of the aggregates, divided by the number of aggregates. The equivalent diameter $\left(D_{\text {eq }}\right)$ was calculated based on area determination using the following equation:

$$
D_{\text {eq }}=2 F_{\text {Cal }} \sqrt{\frac{\text { Area }}{\pi}}
$$

where $\mathrm{F}_{\mathrm{Cal}}$ is the calibration factor ( $\mu \mathrm{m}$ per pixel).

The perimeter was calculated by

$$
P=N_{\text {Per }} \times 1.1222 \times F_{\text {Cal }}
$$

where $N_{\text {Per }}$ is the pixel sum of the object boundaries. The factor 1.1222 was used in order to homogenize the different angles of the filaments. ${ }^{19}$ Other parameters were based on the Feret diameter, 


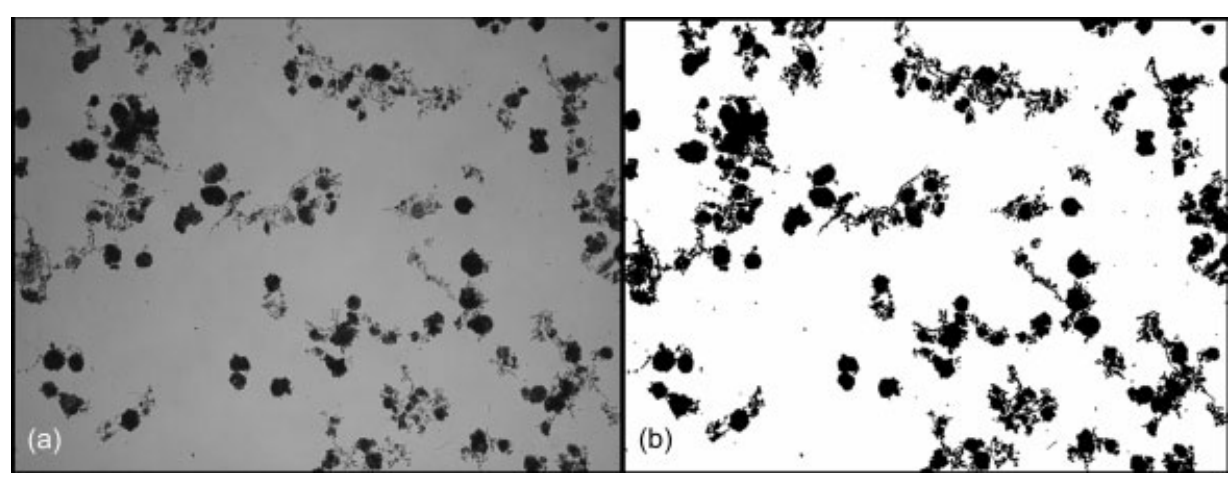

Figure 1. (a) Original image from the activated sludge system with $40 \times$ magnification. (b) Final image of binary aggregates.

which is the maximum distance between two parallel tangents touching opposite borders of the object. ${ }^{20}$ The length of each aggregate was given by the maximum Feret diameter converted to metric units, and the width was given by the minimum Feret diameter converted to metric units. ${ }^{21}$ The extent was calculated as the ratio between the area of an object and its bounding box area. Eccentricity was calculated using the area and the second-order moments $\left(M_{2}\right)$ of an object determined by ${ }^{20}$

$$
\mathrm{Ecc}=\frac{(4 \pi)^{2}\left(M_{2 X}-M_{2 Y}\right)^{2}+4 M_{2 X Y}^{2}}{\text { Area }^{2}}
$$

Convexity is defined as the ratio between the convex perimeter $\left(P_{\text {Conv }}\right)$ and the perimeter $(P)$ of an object based on the following equation:

$$
\text { Conv }=\frac{P_{\text {Conv }}}{P}
$$

Roundness was calculated using the area and the convex perimeter of an object by ${ }^{18}$

$$
\text { Round }=\frac{4 \pi \text { Area }}{P_{\text {Conv }}^{2}}
$$

Compactness was calculated using the area and maximum Feret diameter $\left(F_{\text {Max }}\right)$ of an object based on the following equation: ${ }^{19}$

$$
\text { Comp }=\frac{\sqrt{(4 / \pi)} \text { Area }}{F_{\operatorname{Max}}}
$$

Robustness was given by the following equation:

$$
\text { Robus }=\frac{2 \mathrm{er}_{\mathrm{obj}}}{\sqrt{\text { Area }}}
$$

where $\mathrm{er}_{\mathrm{obj}}$ is the erosion needed to delete an object. ${ }^{20}$

Solidity is defined as the ratio between the object area and the convex envelope area. Finally, the total aggregate area and total aggregate number were determined. ${ }^{21}$

\section{Multivariate statistical techniques}

Partial least squares regression (PLS) is an iterative algorithm that extracts linear combinations of the essential features of the original $\mathbf{X}$ data while modeling the $\mathbf{Y}$ data dependence on the work set, and is therefore well suited for multivariate calibration. ${ }^{22}$ In this method, the latent variables ui (matrix $U$ ) are used for modeling the objects separately in the matrix of $\mathbf{Y}$ dependent data, whereas, the ti variables (matrix $\mathrm{T}$ ) are used for modeling the objects separately in the $\mathbf{X}$ matrix of independent data. The latent variables $U$ and $T$ are the basis of the regression model and are determined by $\mathrm{U}=\mathrm{A} \times \mathrm{T}+\mathrm{E}$ (PLS components matrix $\mathrm{A}$ and error matrix $\mathrm{E})$ in an iterative process with the centered matrices of $\mathbf{X}$ and $\mathbf{Y}$ as starting points. SIMCA 8.0 (Umetrics, Umeå, Sweden) software package was used to perform PLS analysis from the dataset. This software iteratively computes one PLS at a time, that is, one vector each of $X$-scores $(t), Y$-scores $(u)$, weights expressing the correlation between $\mathbf{X}$ and $U(w)$, weights expressing the correlation between $\mathbf{Y}$ and $\mathrm{T}(c)$ and loadings $(p)$. The PLS components are calculated in descending order of importance. This analysis gave relationships between the parameters obtained by image analysis and the classic settling parameters determined using conventional procedures.

\section{RESULTS AND DISCUSSION \\ Effect of salt content on SVI and turbidity}

SVI measurement plays an important role in activated sludge evaluation, representing the morphological and aggregate settling ability. Several studies have already been reported considering the SVI measure on the study of saline effluents, showing that the increase in salt concentration incites physical aggregate modification, ${ }^{23}$ organism plasmolysis, ${ }^{4}$ biomass content reduction, and aggregate size reduction. ${ }^{24}$ Contrasting with SVI, turbidity can reflect the flocculation of non-settling smaller aggregates or dispersed microorganisms. Usually, if microbial aggregates are well flocculated, they will become part of the settling matrix and, if not, they will not settle. High contents of non-settling small aggregates and free bacteria can lead to a high turbidity of the treated effluent. ${ }^{25}$ Fig. 2 illustrates the behavior of turbidity and SVI with the salt content for the current study. The results depicted in Fig. 2 show that SVI decreased with salt content up to $20 \mathrm{~g} \mathrm{~L}^{-1}$, remaining somewhat stable above this value despite the turbid effluent. A gradual increase in turbidity was observed until stabilization at $40 \mathrm{~g} \mathrm{~L}^{-1}$ of salt content. The high turbidity seemed to indicate high suspended solids content, which may have been due to an increase in the content of smaller aggregates (pinpoint flocs) with low density or even dispersed bacteria (smaller than the current methodology cut-off value). The high effluent turbidity may also have been caused by the lack of filamentous organisms, highly sensitive to the presence of $\mathrm{NaCl}$. Some authors have also suggested that the absence of protozoa, responsible for suspended bacteria control, also leads to higher suspended solids in the effluent. ${ }^{25}$ In the present work, after an initial detection during the experiment with $0 \mathrm{~g} \mathrm{~L}^{-1} \mathrm{NaCl}$, the absence of protozoa in activated sludge was observed during the image acquisition step. Given this fact, it could also be expected that, throughout 


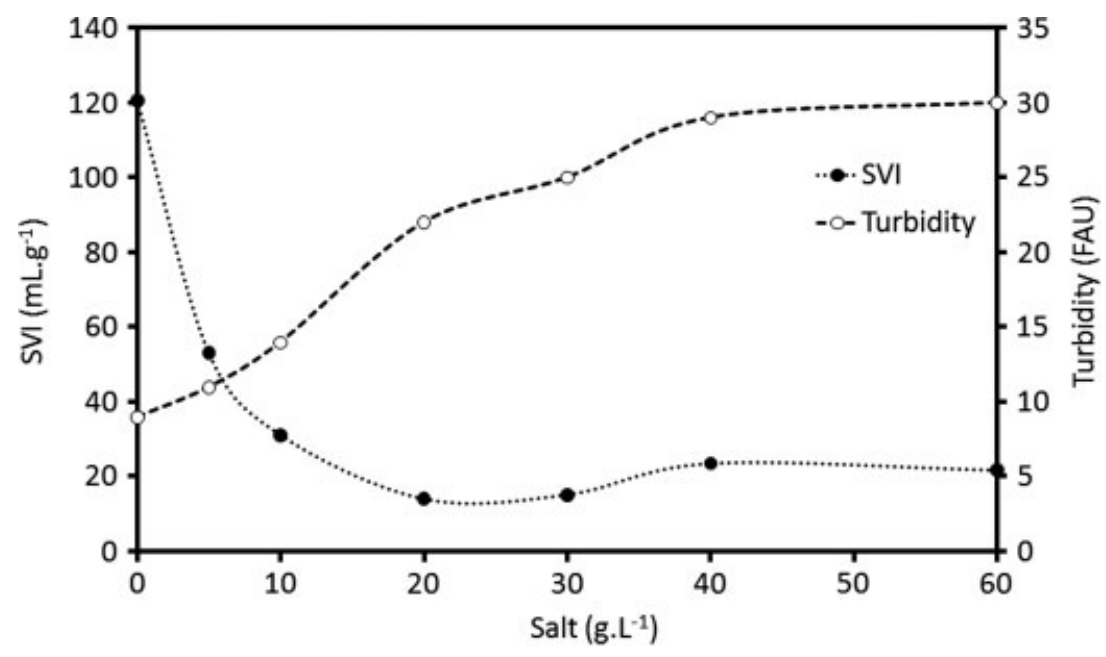

Figure 2. Final sludge volume index (SVI) and turbidity for each $\mathrm{NaCl}$ concentration.

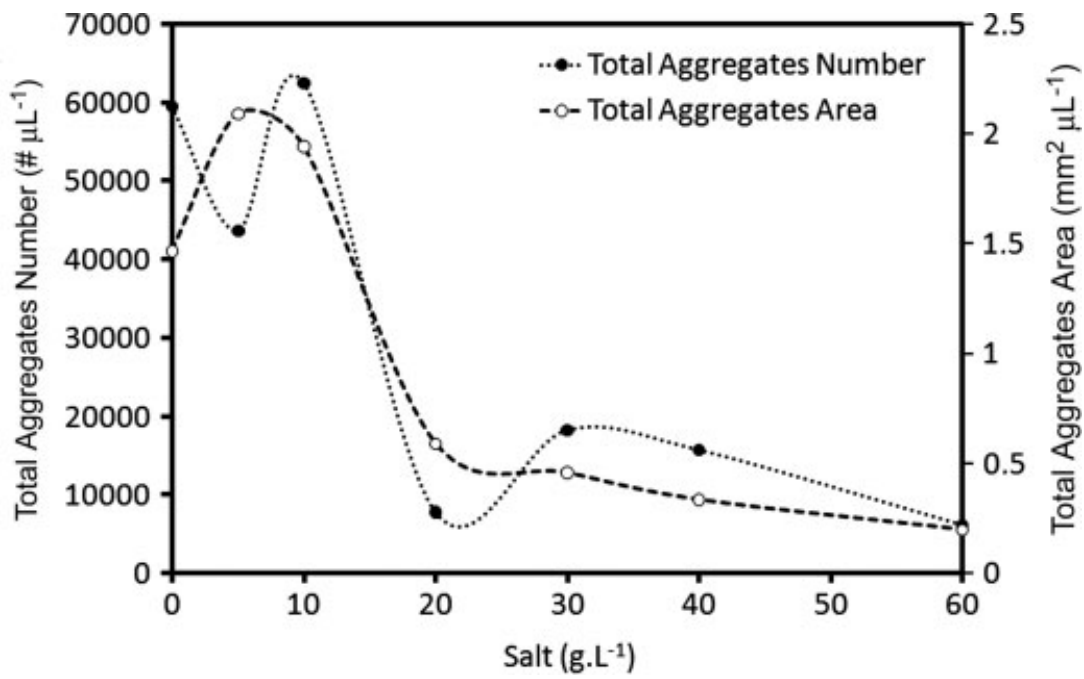

Figure 3. Total aggregate area and total aggregate number profile with $\mathrm{NaCl}$ content.

this experiment, the control that protozoa exert on the bacterial content was lacking, resulting in pinpoint flocs ${ }^{26}$ and dispersed bacteria, which may have been created by the breakage of larger aggregates and leading to an effluent with low SVI but high turbidity.

\section{Aggregate class distribution}

The total aggregate area and total number of aggregates (Fig. 3) revealed an initial disturbance in terms of aggregate contents between $010 \mathrm{~g} \mathrm{~L}^{-1} \mathrm{NaCl}$, followed by a sharp decrease for $20 \mathrm{~g} \mathrm{~L}^{-1}$ of salt and a smooth, gradual decrease thereafter to a final value considerably smaller than the initial one. Therefore, it was found from these results that the increase in salt concentrations, especially from the $5 \mathrm{~g} \mathrm{~L}^{-1} \mathrm{NaCl}$ concentration onwards, incited a continuous and heavy loss of aggregated biomass, as clearly pointed out by the total aggregate area results. The behavior of the total aggregate number is clearly more erratic than the total aggregate area due to the fact that, during the deflocculation phenomena, both free bacteria and aggregates smaller than the methodology cut-off area may be formed, and therefore not accounted for, thus explaining the obtained valley points regarding the 5 and $20 \mathrm{~g} \mathrm{~L}^{-1} \mathrm{NaCl}$ concentrations.

In previous studies, ${ }^{14}$ the usefulness of evaluating aggregates at different size classes was shown. In the present work the aggregates were divided into three classes: equivalent diameter (Deq) below $0.01 \mathrm{~mm}$, Deq between 0.01 and $0.1 \mathrm{~mm}$ and Deq above $0.1 \mathrm{~mm}$. The larger flocs were considered to be normal flocs; the intermediate class was composed of small flocs and the smaller class comprised pinpoint flocs. Structural parameters such as the equivalent diameter of each aggregate class, number and area distributions were evaluated to survey the effect of salt content on aggregate size (Fig. 4).

From Fig. 4, it can be seen that for the larger aggregate class $(>0.1 \mathrm{~mm})$ an initial increase in aggregate size was found for $5 \mathrm{~g} \mathrm{~L}^{-1} \mathrm{NaCl}$, followed by a gradual decrease converging to the initial values for the higher $60 \mathrm{~g} \mathrm{~L}^{-1} \mathrm{NaCl}$ concentration. These results may reflect a degradation of aggregates structure towards looser and therefore larger structures, caused by the initial shock promoted by salt addition. However, as these looser structures are also mechanically more fragile, their continuous erosion could explain the following convergence to the initial size. The results for the intermediate class were quite the opposite, showing 

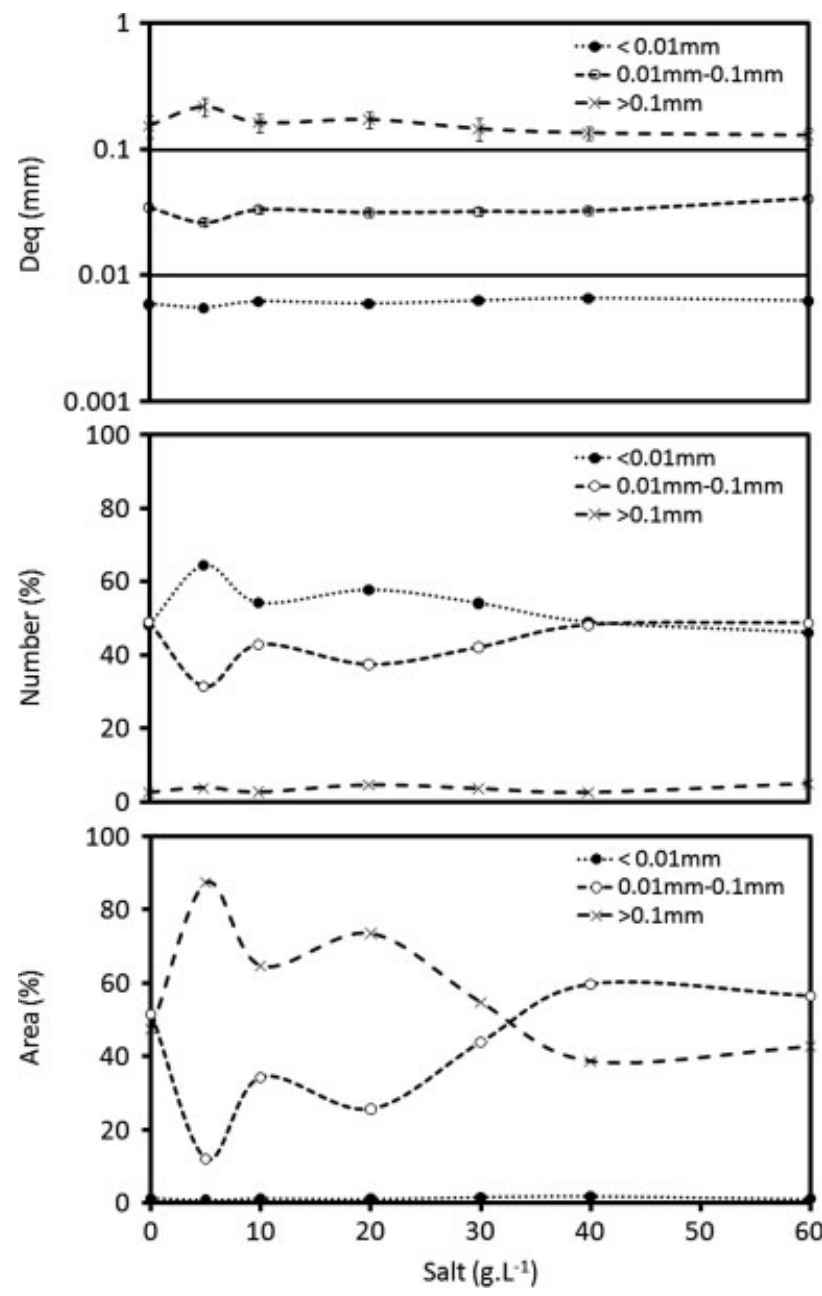

Figure 4. Aggregate equivalent diameter $\left(D_{\mathrm{eq}}\right)$, number and area variation with $\mathrm{NaCl}$ content.

deflocculation provoked by the initial salt addition and their gradual recovery, whereas the size of the pinpoint flocs remained unchanged during the experimental period.

With respect to aggregate number distribution, an initial shift from intermediate to smaller aggregates occurred for the $5 \mathrm{~g} \mathrm{~L}^{-1} \mathrm{NaCl}$ concentration, probably due to a deflocculation phenomenon caused by the initial salt shock, followed by a gradual shift in the opposite sense until the higher $60 \mathrm{~g} \mathrm{~L}^{-1}$ $\mathrm{NaCl}$ concentration, converging to the initial number distribution. Taking into account the overall aggregate number behavior, it is most likely that the intermediate aggregates suffered a deflocculation phenomenon right from the start of the experiment. The percentage of larger aggregates remained approximately stable, thus showing similar behavior to the overall aggregate number. By this means, their absolute value suffered a severe drop from 10 to $20 \mathrm{~g} \mathrm{~L}^{-1}$ salt concentration and a smoother decrease up until the end of the experiment. The behavior of the smaller pinpoint flocs seems to indicate a disaggregation phenomenon on one hand, given their intrinsic low mechanical properties, and the formation of new pinpoint flocs by deflocculation and erosion of larger aggregates on the other. Therefore, the aggregate number distribution results indicate a deflocculation process from the beginning of salt addition, and their convergence to initial values reflects disaggregation of the aggregates mainly from the $5 \mathrm{~g} \mathrm{~L}^{-1}$ concentration onwards.

Finally, analyzing the aggregate area distribution, an initial shift from the intermediate to the larger aggregates occurred for $5 \mathrm{~g} \mathrm{~L}^{-1} \mathrm{NaCl}$, followed by a gradual shift in the opposite direction until the higher $60 \mathrm{~g} \mathrm{~L}^{-1} \mathrm{NaCl}$ concentration, once again converging to the initial area distribution. These results reflect the conclusions above. The initial increase in the larger aggregate area distribution could be established as a direct result of initial shock leading to larger and looser structures and deflocculation of the intermediate aggregates. Furthermore, convergence of the area distribution to the initial values reflects the disaggregation of these large aggregates mainly from the $5 \mathrm{~g} \mathrm{~L}^{-1}$ concentration onwards. It seems clear, therefore, that the initial salt addition led to weakening of the microbial aggregate structure (mainly of the larger and intermediate flocs). Biomass deflocculation seems to be the most suitable explanation, in the sense of the aggregate breakage to form pinpoint flocs as shown by the initial increase of their number distribution. It was also noted that the change to $20 \mathrm{~g} \mathrm{~L}^{-1}$ salt concentration incited a severe disaggregation phenomenon, as shown by the total aggregate number, which could be explained by an insufficient acclimation period. The deflocculation processes described above, apart from originating pinpoint flocs, are very likely to have released high quantities of dispersed bacteria to the bulk, given the weakening of the aggregate structure. Furthermore, this increase in freely dispersed bacteria is in accordance with the high turbidity of the treated effluent. It is also known that conventional organisms such as protozoa are affected by the increment in salt content. ${ }^{27}$ Thus, elimination of the protozoal community from the activated sludge process was expected, and may have allowed for the increase in dispersed bacteria and turbidity levels. In conclusion, a high loss of aggregated biomass could be established, mainly from the $20 \mathrm{~g} \mathrm{~L}^{-1}$ salt concentration, probably due to an insufficient initial acclimation period followed by gradual recovery of the biomass structure, but not content, throughout the remaining experiment.

\section{Morphological parameters}

It is known that the area, equivalent diameter, perimeter, length and width are size-related variables; solidity, roundness, and extent represent the space fulfilling ability; compactness and eccentricity the elongation of the object, convexity the roughness of the object's borders and robustness combine the flocs fulfilling ability and the border's roughness. ${ }^{14}$ In order to establish the best parameters to describe the microbial aggregate morphology and sludge characteristics, a cross-correlation analysis was performed among all the data.

From this cross-correlation analysis it emerged that four of the five parameters representing the aggregate size (area, equivalent diameter, length and width) presented a correlation factor above 0.95. Such was also the case with the elongation parameters (compactness and eccentricity) and two of the three space-fulfilling ability descriptors (solidity and extent). In order to decrease the data matrix in the subsequent SVI multivariable analysis, a partial least squares (PLS) analysis was performed with the SVI as $\mathbf{Y}$ data and each of the two above-mentioned clusters in order to establish the most important parameter within these clusters. The results of the variable importance in the projection (VIP) allowed determination of the most suitable parameters of each cluster to correspond to the $\mathbf{Y}$ data (SVI). For the aggregate size cluster, the VIP results showed that the aggregate average area (1.3911) was the best parameter, whereas for the morphological parameters 
compactness proved to be better suited (1.3014) than eccentricity and solidity better (1.1684) than extent and roundness.

In order to elucidate the parameter relationships PLS regression was performed on this dataset with SVI as $\mathbf{Y}$ variable and as $\mathbf{X}$ variables the single best parameter of each cluster (solidity, total aggregate area, robustness, aggregate average area, convexity and compactness). The results of the variables' importance from the above-mentioned parameters allowed determination of the most suitable parameters to correspond to the $\mathbf{Y}$ data (SVI).

It is known that when the number of $\mathbf{X}$ variables is close to the number of $\mathbf{Y}$ data points the prediction ability is always high and therefore cannot be taken into account. Therefore, and in order to avoid such an effect on the prediction ability of this work (given that only seven SVI data points were available), no more than three parameters were studied for subsequent prediction analysis. The parameter found to contribute most to the SVI (highest VIP value) was convexity, with a value of 1.3962 , followed by robustness (1.2546) and total aggregate area (1.1486).

The PLS regression to estimate the SVI values is presented in Fig. 5 and attained a value for the correlation coefficient of $0.991\left(R^{2} 0.9839\right)$ between the observed and predicted SVI values. Although a good correlation coefficient was obtained, caution is advised given the low number of SVI data points and the proximity of the values.

Keeping in mind the three major morphological aspects (border roughness, fulfilling ability and elongation) represented by the presented morphological data, the best parameter representing each aspect was chosen to elucidate the aggregate changes with salt addition. Therefore, in the PLS analysis results, elongation was found to be better represented by compactness, space-fulfilling ability by solidity, and border roughness by convexity. With a good VIP, however, the robustness parameter conjugates two of the above morphology characterizations: fulfilling ability and border roughness.

Analysis of the most significant morphological parameters ${ }^{13}$ during the experiments, namely convexity, solidity and compactness, revealed the predominance of the larger aggregates $(>0.1 \mathrm{~mm}$ ) of somewhat loose aggregates (low solidity values around 0.55 ), and those that were elongated (low compactness around 0.58 ), and quite rough on the edges (very low convexity values around 0.30$)$. Intermediate aggregates $(0.01-0.1 \mathrm{~mm}$ ) were found to be morphologically similar to what could be expected for smaller flocs (pinpoint flocs): more compact (higher solidity

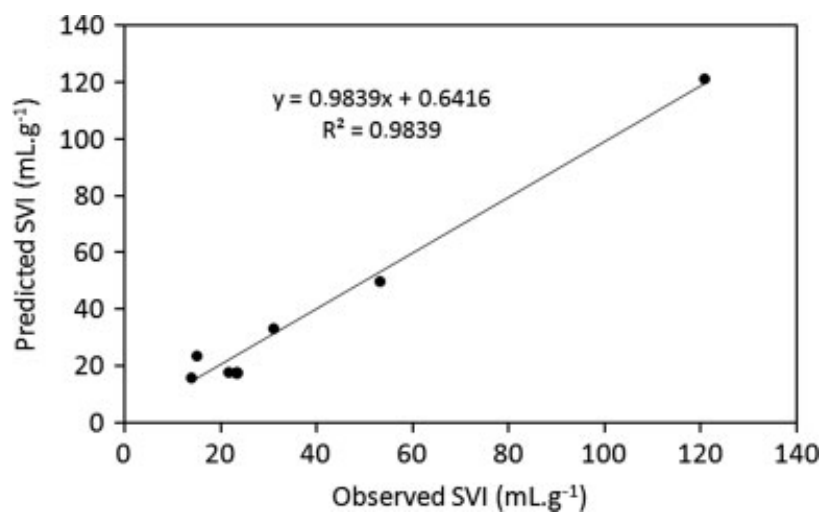

Figure 5. Relationship between predicted and observed SVI after PLS regression with robustness, extent and total aggregate area (linear correlation coefficient $R=0.991$ ). than 0.74), less elongated (higher compactness than 0.68), and smoother (much higher convexity than 0.60 ).

\section{CONCLUSIONS}

It was established that loss of aggregated biomass is due to pinpoint flocs and dispersed bacterial growth, as observed by an increase in effluent turbidity. This effect was more severe after $20 \mathrm{~g} \mathrm{~L}^{-1} \mathrm{NaCl}$ addition, clearly pointing to an insufficient acclimation period of the biomass. The results of aggregate morphological analysis showed the predominance of somewhat loose aggregates, elongated and rough on the edges for larger aggregates and more compact, less elongated and much smoother for the intermediate class. Both aggregate size and morphological analysis seem to point to a biomass deflocculation of intermediate aggregates for $5 \mathrm{~g} \mathrm{~L}^{-1} \mathrm{NaCl}$ addition and of the larger aggregates for $10 \mathrm{~g} \mathrm{~L}^{-1} \mathrm{NaCl}$ addition. With respect to PLS analysis to estimate SVI values, and although a good correlation coefficient was obtained (0.991), caution is advisable given the low number of SVI data points and proximity of the values.

\section{ACKNOWLEDGEMENTS}

The authors are grateful to the ALFA cooperation project BIEURAM III (European Commission) between Universidade do Minho and Universidade Federal do Rio de Janeiro. MAZ Coelho acknowledges CNPq (Brazil) for financial support for this work. We also acknowledge financial support to Daniela Mesquita through project $\mathrm{POCl} / \mathrm{AMB} / 57069 / 2004$ provided by Fundação para a Ciência e Tecnologia (Portugal).

\section{REFERENCES}

1 Moussa MS, Sumanasekera DU, Ibrahim SH, Lubberding HJ, Hooijmans CM, Gijzena HJ, et al, Long term effects of salt on activity, population structure and floc characteristics in enriched bacterial cultures of nitrifiers. Water Res 40:1377-1388 (2006).

2 Rene ER, Kim SJ and Park HS, Effect of COD/N ratio and salinity on the performance of sequencing batch reactors. Bioresour Technol 99:839-846 (2007).

3 Uygur A, Specific nutrient removal rates in saline wastewater treatment using sequencing batch reactor. Process Biochem 41:61-66 (2006).

4 Uygur A and Kargi F, Salt inhibition on biological nutrient removal from saline wastewater in a sequencing batch reactor. Enzyme Microbial Technol 34:313-318 (2004).

5 Intrasungkha N, Keller J and Blackall L, Biological nutrient removal efficiency in treatment of saline wastewater. Water Sci Technol 39:183-190 (1999).

6 Panswad T and Anan C, Impact of high chloride wastewater on an anaerobic/anoxic/aerobic process with and without inoculation of chloride acclimated seeds. Water Res 33:1165-1172 (1999).

7 Ekama GA, Barnard JL, Günthert FW, Krebs P, McCorquodale JA, Parker DS, et al, Secondary Settling Tanks: Theory, Modelling, Design and Operation. IAWQ Scientific and Technical Reports, IAWQ, London (1997).

$8 \mathrm{LiX}$ and Yuan Y, Settling velocities and permeabilities of microbial aggregates. Water Res 36:3110-3120 (2002).

9 Ginoris Y, Leite SGF and Coelho MAZ, Activated sludge morphology characterization through image analysis procedure. Braz J Chem Eng 23:319-330 (2006).

10 Grijspeerdt K and Verstraete W, Image analysis to estimate the settleability and concentration of activated sludge. Water Res 31:1126-1134 (1997).

11 Jenné R, Banadda EN, Smets IY, Deurinck J and Van Impe JF, Detection of filamentous bulking problems: developing an image analysis system for sludge composition monitoring. Microsc Microanal 13:36-41 (2007). 
12 Jenné R, Banadda EN, Smets IY and Van Impe JF, Monitoring activated sludge settling properties using image analysis. Water Sci Technol 50:281-285 (2004).

13 Amaral AL, Image analysis in biotechnological processes: applications to wastewater treatment. [Online]. PhD thesis, University of Minho, Portugal (2003). Available: http://hdl.handle.net/1822/4506 [14 October 2008].

14 Amaral AL and Ferreira EC, Activated sludge monitoring of a wastewater treatment plant using image analysis and partial least squares regression. Anal Chim Acta 544:246-253 (2005).

15 Coelho MAZ, Russo C and Araújo OQF, Optimization of a sequencing batch reactor for biological nitrogen removal. Water Res 34:2809-2817 (2000).

16 Seixo J, Varela MH, Coutinho JAP and Coelho MAZ, Influence of C/N ratio on autotrophic biomass development in a sequencing batch reactor. Biochem Eng J 21:131-139 (2004).

17 Souza SM, Araujo OQF and Coelho MAZ, Model-based optimization of a sequencing batch reactor for biological nitrogen removal. Bioresour Technol 99:3213-3223 (2008)

18 APHA, AWWA, WPCF, Standard Methods for the Examination of Water and Wastewater. American Public Health Association, Washington, DC (1989)
19 Walsby AE and Avery A, Measurement of filamentous cyanobacteria by image analysis. J Microbiol Methods 26:11-20 (1996).

20 Glasbey CA and Horgan GW, Image Analysis for the Biological Sciences. Wiley, Chichester (1995).

21 Russ CR, The Image Processing Handbook. CRC Press, Boca Raton, FL (1995).

22 Einax JW, Zwanziger HW and Geiss S, Chemometrics in Environmental Analysis. VCH Verlagsgesellshaft, Weinheim (1997).

23 Moon BH, Seo GT, LeeTS, Kim SS and Yoon CH, Effects of salt concentration on floc characteristics and pollutants removal efficiencies in treatment of seafood wastewater by SBR. Water Sci Technol 47:65-70 (2002).

$24 \mathrm{NgHY}$, Ong SL and $\mathrm{Ng} \mathrm{WJ}$, Effects of sodium chloride on the performance of a sequencing batch reactor. J Environ Eng 131:1557-1564 (2005)

25 Campos JL, Mosquera A, Sánchez M, Méndez M and Lema JM, Nitrification in saline wastewater with high ammonia concentration in an activated sludge unit. Water Res 36:2555-2560 (2002).

26 Bitton G, Wastewater Microbiology. Wiley-Liss, New York (1994).

27 Salvadó H, Mas M, Menéndez S and Gracia MP, Effects of shock loads of salt on protozoan communities of activated sludge. Acta Protozool 40:177-185 (2001). 